ARTICLE

Received 18 Mar 2014 | Accepted 4 Jun 2014 | Published 8 Jul 2014

DOI: $10.1038 /$ ncomms5302

\title{
Enantioselective control of lattice and shape chirality in inorganic nanostructures using chiral biomolecules
}

Assaf Ben-Moshe', Sharon Grayer Wolf ${ }^{2}$, Maya Bar Sadan ${ }^{3}$, Lothar Houben ${ }^{4}$, Zhiyuan Fan ${ }^{5}$, Alexander O. Govorov ${ }^{5} \&$ Gil Markovich ${ }^{1}$

A large number of inorganic materials form crystals with chiral symmetry groups. Enantioselectively synthesizing nanostructures of such materials should lead to interesting optical activity effects. Here we report the synthesis of colloidal tellurium and selenium nanostructures using thiolated chiral biomolecules. The synthesis conditions are tuned to obtain tellurium nanostructures with chiral shapes and large optical activity. These nanostructures exhibit visible optical and chiroptical responses that shift with size and are successfully simulated by an electromagnetic model. The model shows that they behave as chiral optical resonators. The chiral tellurium nanostructures are transformed into chiral gold and silver telluride nanostructures with very large chiroptical activity, demonstrating a simple colloidal chemistry path to chiral plasmonic and semiconductor metamaterials. These materials are natural candidates for studies related to interactions of chiral (bio)molecules with chiral inorganic surfaces, with relevance to asymmetric catalysis, chiral crystallization and the evolution of homochirality in biomolecules.

\footnotetext{
${ }^{1}$ Raymond and Beverly Sackler Faculty of Exact Sciences, School of Chemistry, Tel Aviv University, Tel Aviv 69978, Israel. ${ }^{2}$ Electron Microscopy Unit, Weizmann Institute of Science, Rehovot 76100, Israel. ${ }^{3}$ Department of Chemistry, Ben Gurion University of the Negev, Beer Sheba 84105, Israel. ${ }^{4}$ Ernst Ruska-Centre for Microscopy and Spectroscopy with Electrons, Forschungszentrum Jülich GmbH, 52425 Jülich, Germany. ${ }^{5}$ Department of Physics and Astronomy, Ohio University, Athens, Ohio 45701, USA. Correspondence and requests for materials should be addressed to G.M. (email: gilmar@post.tau.ac.il).
} 
C hirality is the geometric property of structures that are non-superimposable with their mirror images. This characteristic is a fundamental aspect of biomolecules such as proteins and nucleic acids, rendering specificity in biomolecular recognition and structure-function relations. Although chiral crystals were considered as sources for the generation of homochirlity in biomolecules ${ }^{1,2}$, the opposite effect of biomolecules inducing homochirality in chiral crystals was not thoroughly addressed.

Chiral systems also show unique optical (chiroptical) effects when interacting with polarized electromagnetic radiation. The effect commonly measured is circular dichroism (CD), the differential absorption of left and right circularly polarized light by chiral molecules and materials ${ }^{3}$. Unlike organic molecules, where the chiroptical effects are typically small at visible wavelengths, larger inorganic systems (roughly over ten nanometres to several hundreds of nanometres) may show stronger chiroptical effects ${ }^{4-6}$. Several mechanisms were suggested for induction of chiroptical phenomena in such systems (both metallic and semiconducting). In plasmonic nanosystems the dipolar nearfield interaction ${ }^{7}$, or a far-field electromagnetic coupling 8,9 between a metal nanoparticle and a chiral molecule could lead to induction of $\mathrm{CD}$ in the plasmon resonance as well as enhancement of the molecular CD by the near-field coupling ${ }^{10,11}$. These effects, which may be useful for biomolecular sensing 9,12 and are fundamentally interesting, are nevertheless limited in magnitude. Stronger effects could be obtained in systems of achiral nanoparticles arranged in a chiral superstructure with strong interparticle interaction ${ }^{13-16}$. Recently, Kotov and colleagues ${ }^{17}$ demonstrated that the chiroptical activity of a chiral particle dimer is intense enough to monitor on a single nanoparticle level. The most intuitive approach to obtain large chiroptical activity is by forming nanoparticles with an actual chiral shape such as a gammadion or a helix. This has been achieved by lithographic methods ${ }^{18-20}$. Recently, there have been several reports on the preparation of chiral inorganic nanostructures through non-lithographic methods: Liedl and colleagues $^{21}$ formed chiral plasmonic shapes using DNA Origami. Fischer and colleagues ${ }^{22}$ used a unique setup for vapour deposition of inorganic materials with excellent control over shape, chirality and material composition. Kotov and colleagues $^{23}$ formed chiral metal nanoshells on initially achiral $\mathrm{ZnO}$ nanopillars by vacuum deposition at varying angles. Reports on formation of chiral shapes with enantiomeric excess for semiconductor $^{24}$ and mesoporous ${ }^{25,26}$ materials also exist. In all these demonstrations the lattice symmetry of the crystal remains achiral and optical activity is obtained through the shape of the whole nanostructure.

Many inorganic materials crystallize with a chiral lattice symmetry, and enantioselective nucleation and growth chemistry could be used to obtain non-racemic mixtures of nanocrystals of these materials. Saeva et al. ${ }^{27}$ synthesized micron-scale selenium (Se) crystals in a chiral cellulose matrix. Trigonal Se (as well as tellurium (Te)) crystallizes in the chiral space group $P 3_{1} 21$ (or its enantiomorph $P 3_{2} 21$ ). In ref. 27 , the authors managed to get a sample with an enantiomeric excess of Se crystallites and measure $\mathrm{CD}$ at the electronic transitions of Se, but without structural characterization. The chiroptical activity was attributed to the lattice chirality of the crystals. The $\mathrm{CD}$ measurements in that work were probably strongly affected by light scattering and possible birefringence of the polymer matrix holding the Se microstructures. At the nanoscale, the concept was first demonstrated by us for cinnabar $(\alpha-\mathrm{HgS})$ nanocrystals that crystallize in the same chiral space group as Se and $\mathrm{Te}^{28}$. In that work, both enantiomers could be obtained, but the demonstration was still limited to lattice chirality only, and the obtained nanocrystal shape was achiral.
In the present work, chiral nanocrystals of Te and Se with a variety of shapes, which exhibit chiroptical activity, are obtained. Previous reports on the synthesis of Te and Se nanomaterials ${ }^{29-31}$ did not relate to the chirality or chiroptical activity of the nanostructures, and there were no attempts to direct their handedness. Here, in addition to lattice chirality of nanocrystals, the overall shape of Te nanocrystals is made chiral, to achieve a new level of control over chirality. Consequently, chirality can be controlled at two hierarchies, atomic lattice and mesoscale shape. These nanocrystals grow by a unique self-assembly process. A model simulation shows that the dominant chiroptical activity of these Te nanostructures comes from the shape chirality, rather than the chiral atomic arrangement. Furthermore, the reactivity of Te towards various chemical transformation processes allows templating other materials into chiral shapes.

\section{Results}

Synthesis of the Te and Se nanostructures. Te and Se precursors were reduced in the presence of a large concentration of thiolated chiral biomolecules such as cysteine, penicillamine and glutathione, where the thiol group strongly binds to Te or Se. These biomolecules proved to be very efficient reducing agents through the thiol group oxidation, as previously discussed for the case of $\mathrm{Te}(\mathrm{IV})$ species $^{32,33}$. An additional reducing agent, hydrazine, was added, in some cases, immediately before or after the fast reduction by the biomolecule. Shape control was achieved by varying the type of biomolecule used, order of reagent addition, reaction time and temperature.

All the different samples obtained are depicted in the transmission electron microscopy (TEM) images in Fig. 1 (Te samples Fig. 1a-e and Se Fig. 1f, see also Supplementary Fig. 1). For all the samples, high-resolution TEM images and electron diffraction patterns verified the formation of the chiral trigonal crystal phase of these materials (see Supplementary Figs 2-5). All the syntheses were highly reproducible and repeated procedures for each sample always resulted in the same shape of particles and same CD spectra. In particular, the synthesis of the most intriguing sample 5 was repeated about 50 times reproducibly.

The difference in product shape/size on using different biomolecules might be attributed to the exact nature of reduction of cationic $\mathrm{Te}$ species by thiolates. As the $\mathrm{Te}$ reduction mechanism proceeds through initial formation of a complex of $\mathrm{Te}(\mathrm{IV})$ with four thiolate molecules ${ }^{32}$, the kinetics of the reaction should depend on the structure of the ligand molecule, which affects the geometry and stability of this complex, and the way it allows assembly of Te atom dimers or oligomers. Reduction of $\mathrm{Te}(\mathrm{IV})$ by hydrazine was much slower than by the biomolecules and it is not dominant in early stages of the reduction process. This is proven by replacing hydrazine with non-reducing molecules containing amine groups that produce the same results (see Supplementary Fig. 6). Hydrazine addition before the reducing biomolecule caused a slower reduction by the biomolecule; hence, it was used to modify the kinetics of the reaction.

Chiroptical properties. Absorption and CD spectra of all the samples appear in Fig. 2. As demonstrated in Fig. 2c, the CD could be reversed by choice of the opposite enantiomer of the biomolecule, indicating the formation of opposite crystal enantiomers. As the chiral biomolecules only absorb in the far ultraviolet region $(<250 \mathrm{~nm})$, the optical activity can only be assigned to excitation of states in the inorganic material. The intensity of chiroptical effects is usually evaluated by considering a normalized quantity, the dissymmetry factor, defined as the CD divided by absorption: $g=\frac{\Delta \mathrm{A}}{\mathrm{A}}=\frac{\Delta \varepsilon}{\varepsilon}$. This eliminates concentration and 

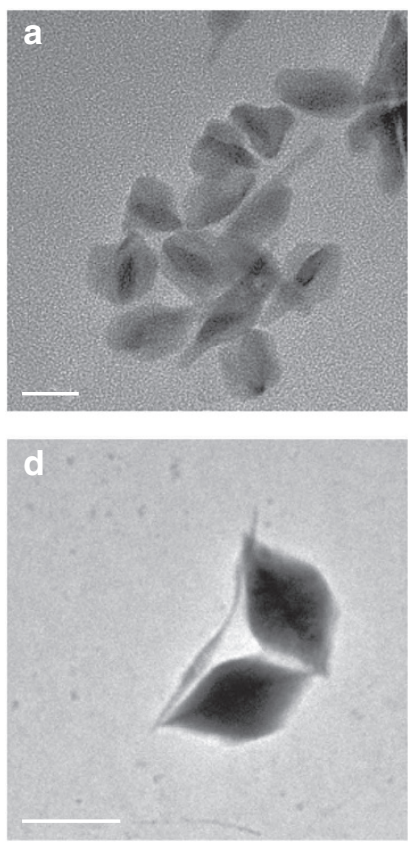
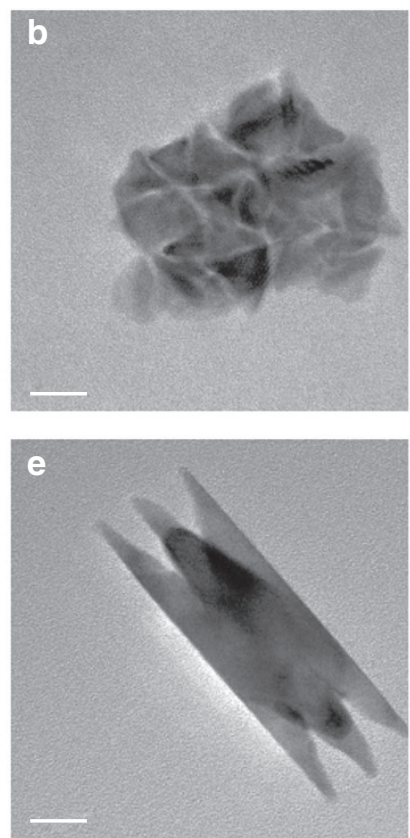
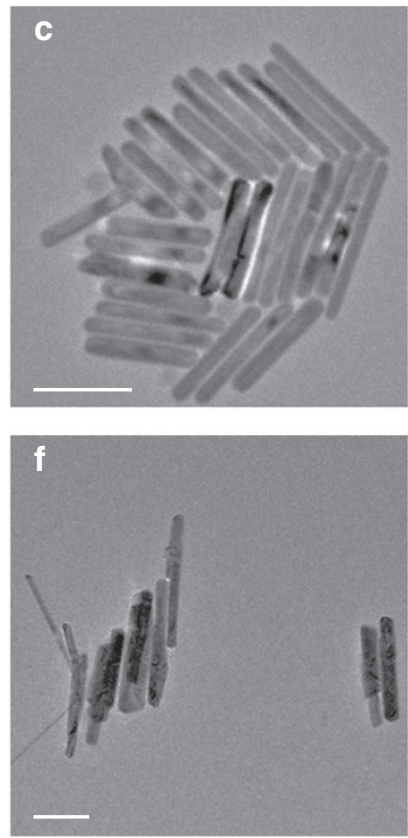

Figure 1 | TEM images of tellurium and selenium nanocrystals produced by different procedures. (a,b) Tellurium nanocrystals (sample 1), obtained with glutathione without hydrazine. Scale bar, $20 \mathrm{~nm}$. In b, the nanocrystals are packed closely, and aligned with their long axis perpendicular to the surface, exhibiting the trigonally symmetric cross section. (c) Long tellurium nanorods (sample 2), obtained with glutathione and hydrazine. Scale bar, $100 \mathrm{~nm}$. (d) Tellurium nanocrystals (samples 3-4) obtained with L- or D-penicillamine and hydrazine. Scale bar, $200 \mathrm{~nm}$. (e) Tellurium nanocrystal (sample 5), obtained with hydrazine and glutathione added in a reversed order of sample 2. Scale bar, 20 nm. (f) Selenium nanocrystals obtained with cysteine. Scale bar, $200 \mathrm{~nm}$.

extinction coefficient dependence when comparing different samples.

For the Se nanocrystals, the extinction seemed to be dominated by scattering due to the relatively large size of the nanorods, and the peak dissymmetry value extracted from Fig. $2 c$,d was on the order of 0.002 .

Most of the Te samples had an observable CD peak in the nearultraviolet region $(\sim 350 \mathrm{~nm})$, indicating enantiomeric selectivity in the synthesis of the Te nanocrystals. Apart from the smallest particles in sample 1 (Fig. 1a), the other samples had strong extinction peaks at different wavelength in the visible range (between $\sim 500$ and $600 \mathrm{~nm}$ ). However, only sample 5 (Fig. 1e) exhibited strong optical activity associated with its visible extinction peaks. Sample 2, in spite of strong visible extinction, exhibited weak CD activity across the near-ultraviolet-visible spectrum. There may be two possible explanations for this finding: First, at these specific conditions, enantiomeric excess achieved in the synthesis is small. Second, electronic transitions in $\mathrm{Te}$ across the visible spectrum are characterized by small $\Delta \varepsilon$ values. The second option is supported by the following results: the peak dissymmetry value of sample 5 exceeds 0.003 , which is much larger than the other Te samples as well as most of past results on inorganic colloids. The intense chiroptical activity of sample 5 in comparison with other Te samples calls for consideration of an additional factor other than the chiral lattice symmetry: shape chirality. The shape of the particles from sample 5 was characterized using scanning TEM (STEM) tomography.

Electron tomography. Figure $3 \mathrm{a}, \mathrm{b}$ depict these particles as observed in STEM mode with a dark-field detector. Figure 3c-e display snapshots from the three-dimensional (3D) tomographic reconstruction at different orientations (see also Supplementary Movie 1) of the particle in Fig. 3b. The basic shape of the nanoparticles is approximately trigonal prism. The handedness of each particle can be determined when observing the side faces, where there are twisted ridges crossing diagonally between vertices at the two opposite ends of the prisms.

Nanocrystals obtained with D-glutathione exhibited similar but more deformed shapes, as well as the opposite handedness and $\mathrm{CD}$ with a smaller magnitude and slightly different lineshape (see Supplementary Fig. 7). The inexact mirror image CD lineshape and particle shape are due to the lower purity of the D-form of glutathione (nominally 95\%) that was obtained synthetically, relative to L-glutathione (98\%). In contrast to this, all samples prepared with penicillamine or cysteine (for which both enantiomers are available commercially at same levels of purity) showed an exact mirror image $\mathrm{CD}$ behaviour for opposite enantiomers.

Evolution of Te nanostructures with chiral shape. The mechanism of growth of these shapes was examined in a series of syntheses and TEM experiments, where the growth of these structures was arrested at various stages of the evolution of the trigonal shapes. The reaction was quenched at each interval by mixing a fraction that is removed from the synthesis with a concentrated solution of SDS at room temperature (1:1 volume ratio of sample and $100 \mathrm{mM}$ SDS solution).

As shown in Fig. 4a (and Supplementary Fig. 8), these nanostructures seem to be forming by coalescence of small clusters. The smaller shapes shown in Fig. 4a (developed after $2 \mathrm{~min}$ of reaction) had an average length of $70 \pm 20 \mathrm{~nm}$. The particles of sample 5 ( $15 \mathrm{~min}$ reaction) were the next intermediate stage observed (Figs 1e and $4 \mathrm{~b}$ ). These particles had an average length of $140 \pm 25 \mathrm{~nm}$. These further grew to $\sim 200$-nm-long hexagonal tubes depicted in Fig. 4c $(20 \mathrm{~min})$. After reacting for $1 \mathrm{~h}$, longer tubes of $\sim 300 \mathrm{~nm}$ and different morphology were obtained (Fig. 4d). The trigonal and hexagonal cross sections 

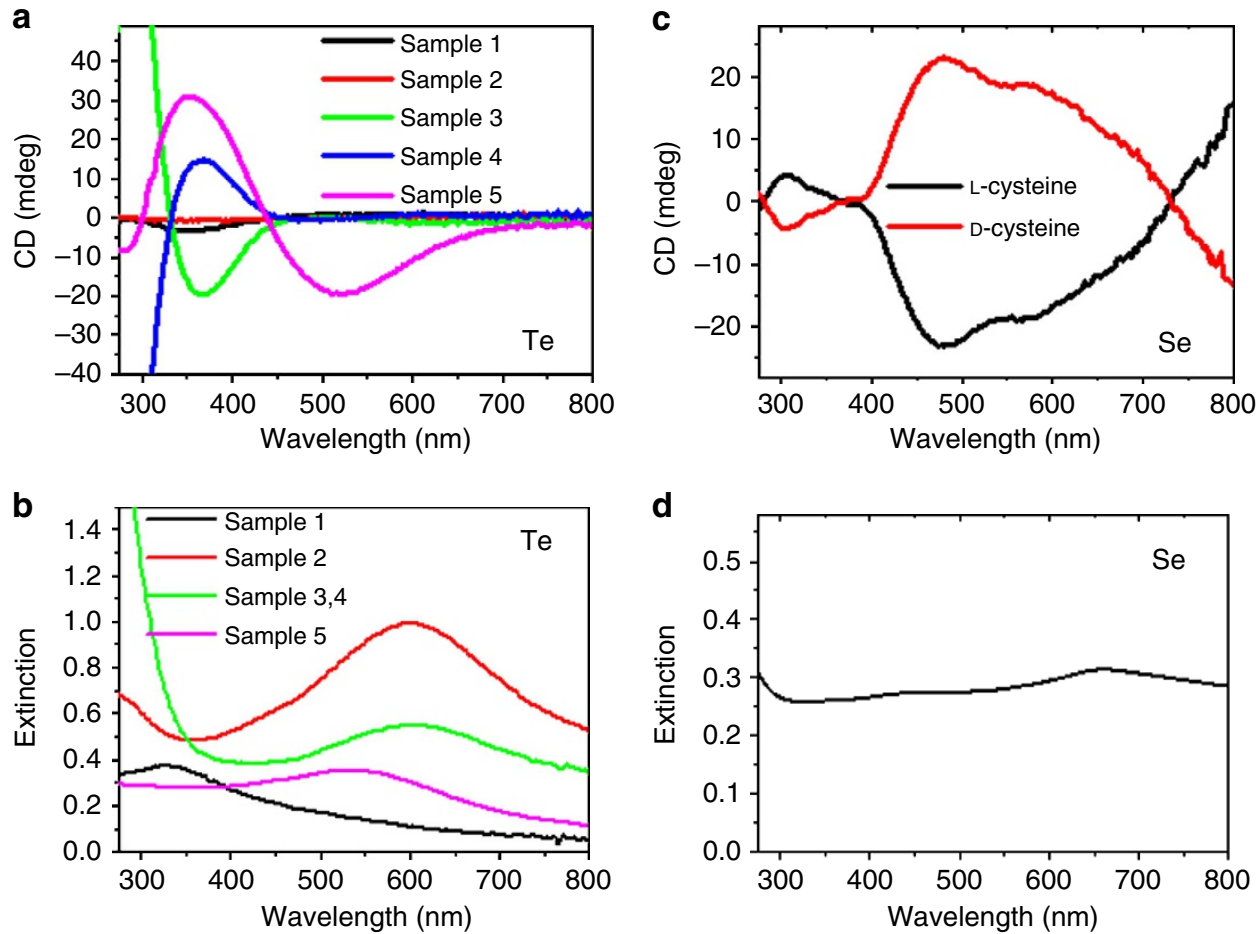

Figure 2 | CD and absorption spectra. CD and absorption spectra of tellurium (a,b) and selenium (c,d) samples. Tellurium sample 5 exhibits dissymmetry values much larger than for other samples of tellurium exceeding 0.003 . For selenium, $g$-values exceed 0.002 .
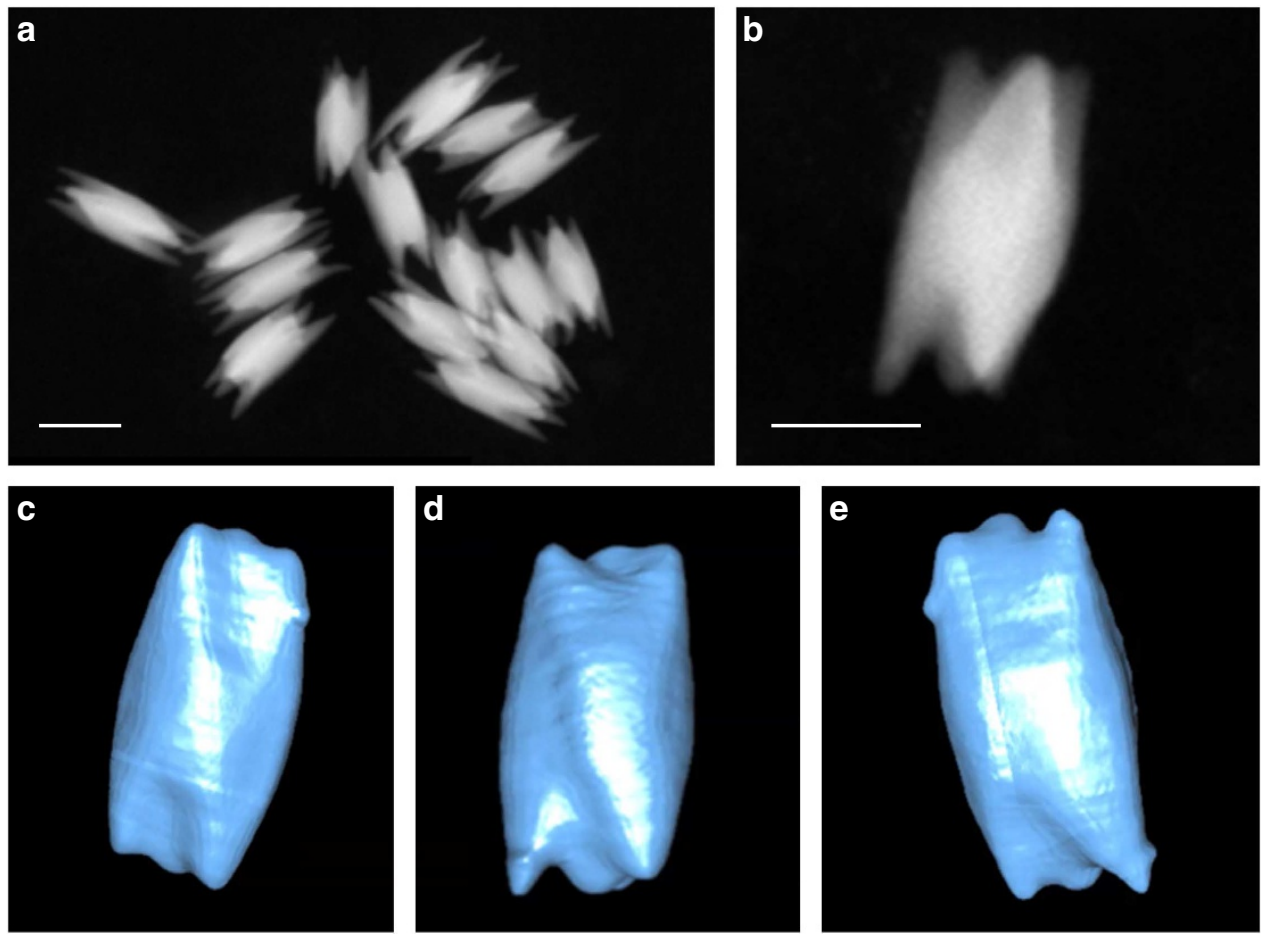

Figure 3 | Dark-field STEM imaging and tomography of tellurium sample 5. (a,b) STEM images at two magnifications. (c-e) Tomographic reconstruction of the particle in $\mathbf{b}$, as observed from three different orientations. Chirality is observed in the twisted ridges crossing diagonally between vertices at the two ends, always stretching from bottom right to top left. Scale bars, $100 \mathrm{~nm}$.

evolving in this process are well observed for particles in dense samples that agglomerate and orient perpendicular to the carbon support film (Fig. 4e,f-corresponding to the samples shown in Fig. 4a,c).
The self-assembly process that is observed at the initial stages of this reaction is intriguing. Recently, Viedma et al. ${ }^{34}$ reported a mechanism for growth of chiral crystals through 'enantiomerspecific oriented attachment'. It seems that in the Te structures 

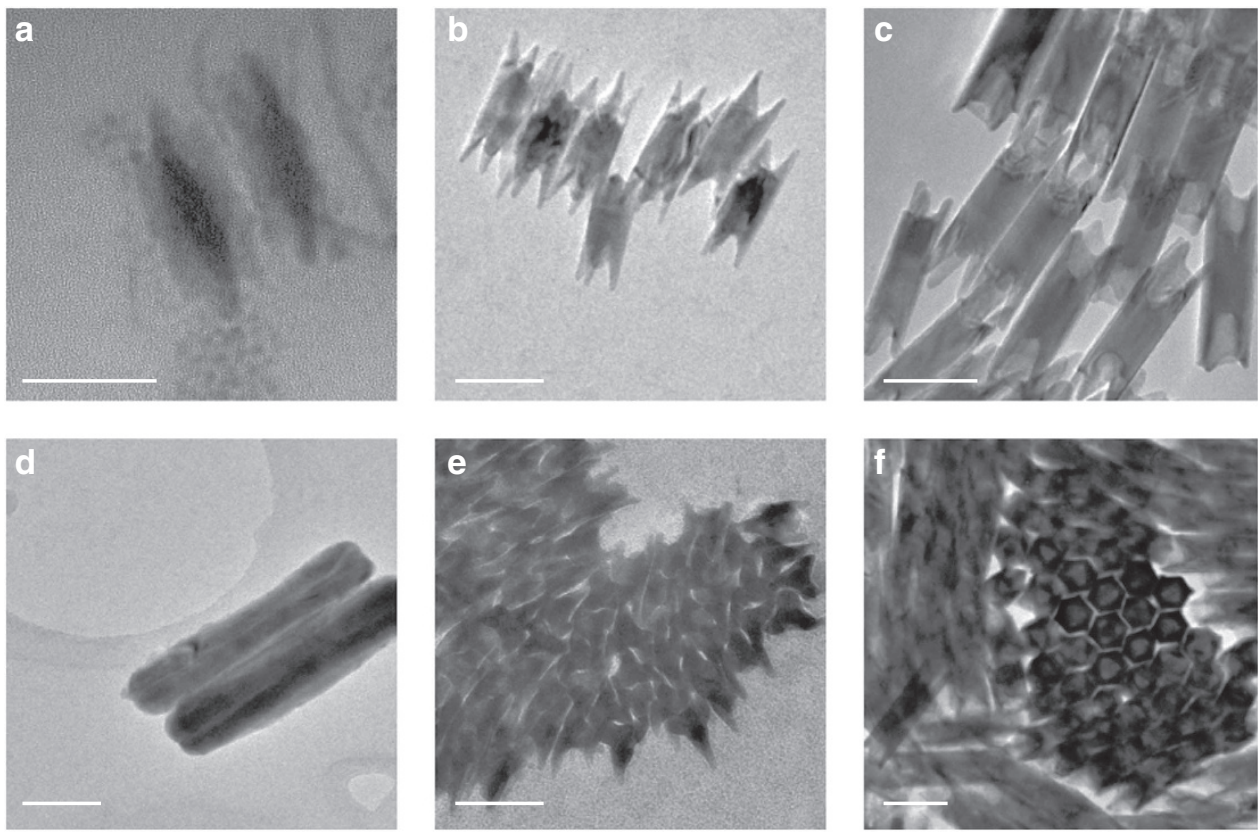

Figure 4 | TEM images of nanocrystals of sample 5 showing evolution over time. (a) Small nanocrystals are initially formed with very small clusters added to the structure. Scale bar, $50 \mathrm{~nm}$. (b) Larger nanocrystals are viewed after another $13 \mathrm{~min}$ of reaction. Scale bar, $100 \mathrm{~nm}$. (c) These evolve into fractured hexagonal tubes. Scale bar, $100 \mathrm{~nm}$. (d) Eventually long hollow tubes are formed. Scale bar, $100 \mathrm{~nm}$. (e,f) Images of nanocrystals from the same samples as in $\mathbf{a}$ and $\mathbf{c}$, respectively, when arranged with the long axis perpendicular to the surface in dense arrays. Scale bar, $100 \mathrm{~nm}$. The transition from trigonal to hexagonal cross section is observed.

presented here, such a process is taking place at the nanoscale, where initially formed chiral atomic clusters attach in an oriented way to form a chiral nanocrystal. It can be seen that the shapes of the evolving Te nanostructures are generally quite complex. The sensitivity of the shape to the particular structure of the chiral thiolated surfactant molecules further strengthens the hypothesis of growth by oriented attachment of surfactant-coated atomic clusters. However, it is still a puzzle how these local attachment effects would lead to shape chirality on a larger $(10-100 \mathrm{~nm})$ scale. Local strain effects caused by the chiral surfactant packing at the surface might be responsible for chiral shape distortions.

Figure 5 displays the absorption and CD spectra of aqueous dispersions of the series of chiral shapes shown in Fig. 4. The first interesting observation, seen in Fig. $5 \mathrm{a}$, is of a red shift between $450-750 \mathrm{~nm}$ in the major absorption/extinction feature with increase in particle dimension (see also Supplementary Fig. 9).

Model electromagnetic calculations. As seen in Fig. 5b, the spectral shift is reproduced by a discrete dipole approximation (DDA) calculation ${ }^{35}$ of the extinction using the bulk $\mathrm{Te}$ dielectric constant ${ }^{36}$. The DDA method is very convenient for computational studies of CD signals, as it permits calculations of extinction with a very high precision. For our goal, we need a high precision, as CD signals are typically much weaker than extinction coefficients. In a recent publication, Fan et al. ${ }^{37}$ have demonstrated that the DDA method is suitable for calculations of weak chiroptical signals of plasmonic nanostructures.

The shape of the particle used in the model was similar to the experimental shapes obtained from the tomogram and the dimensions of the model shape were adjusted according to the TEM measurements. The shift in extinction spectra with size is due to length-dependent electromagnetic resonance modes of these shapes. As Te exhibits a continuum of absorbing states throughout the near-ultraviolet-visible-near-infrared range, those electromagnetic resonances enhance the extinction of the nanoparticles at the resonance wavelengths. In addition, an absorption peak at $300-350 \mathrm{~nm}$, which does not shift with shape or size change, is observable for the larger nanostructures (where there is no spectral overlap with the electromagnetic resonance mode), both in the experimental and simulated absorption spectra of Fig. 5a,b. Thus, it seems that the near-ultraviolet transition around $300-350 \mathrm{~nm}$ is associated with localized Te states and carries a weak CD signal, which is related to the chiral atomic configuration.

The CD spectra of the chiral particle series displayed in Fig. $5 c$ shows that the $\mathrm{CD}$ lines also shift with size as observed with the absorption spectra. The DDA simulated CD dissymmetry factor spectra for two different sizes, plotted in Fig. 5d together with experimental dissymmetry spectra of nanostructures of similar sizes, are only based on the shape chirality and ignore the atomic scale chirality. Considering the rough approximation of shape and size, the simulated spectra agree well with the experimental dissymmetry factors both in wavelength and intensity. The fit between the single particle (100\% enantiomeric excess) simulated $\mathrm{CD}$ amplitude and experimental CD amplitude serves as an indication that at least for sample 5, the enantiomeric excess is high, although it is not yet possible to give an exact value. For nanocrystals with chiral lattice and an achiral shape, estimation of enantiomeric excess is currently an unresolved challenge. For the earlier case of $\alpha-\mathrm{HgS}^{28}$, where data from bulk was available regarding $\mathrm{CD}$ strength, the enantiomeric purity was estimated to be very high under similar synthetic conditions as the current work. However, it is not clear that all materials will yield similar enantioselectivity in the synthesis, and future development of a method to determine enantiomeric excess in these systems, in a more general and exact way, would be indispensable.

The optical response of the Te nanostructures is quite unique relative to other colloidal nanomaterials. Although in wide gap semiconductors there are quantum size effects governing the 


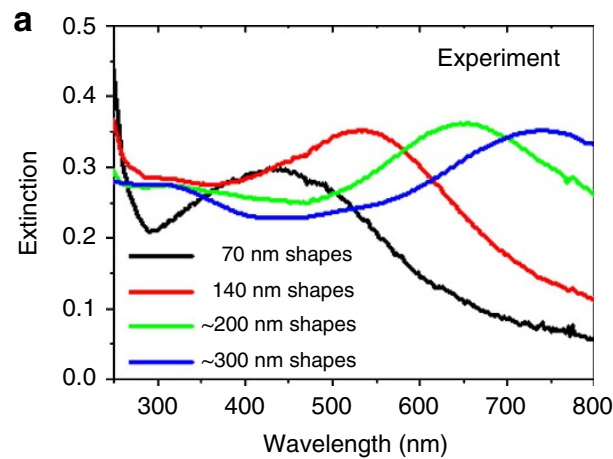

b

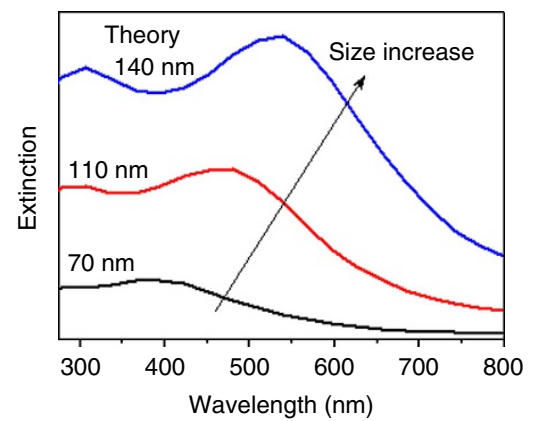

e
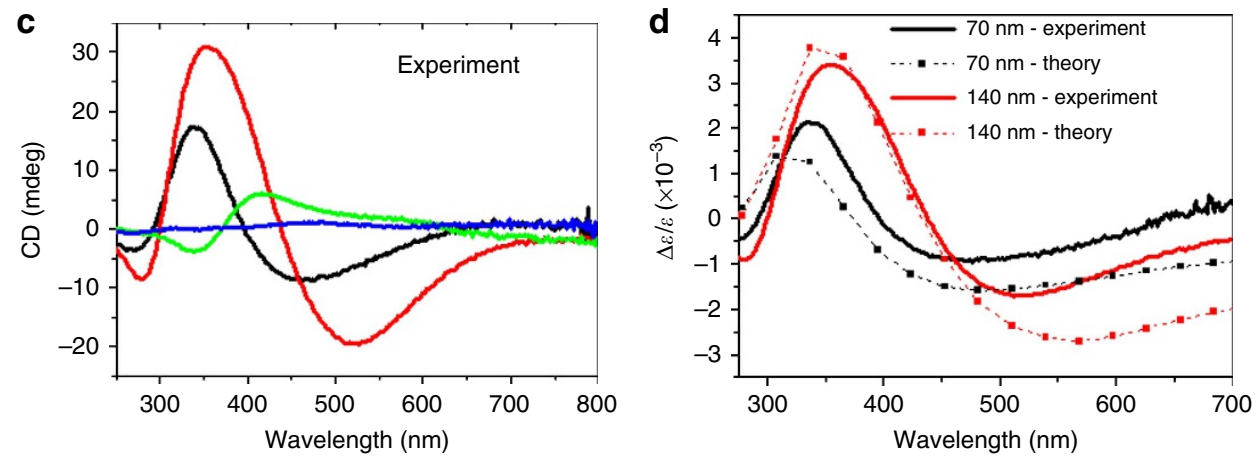

Figure 5 | Absorption and CD activity of chiral shapes of tellurium. Experimental (a) and simulation (b) absorption spectra of samples displayed in Fig. 4. Red shift of the peak position with size is observed. (c) Experimental CD of samples. (d) Experimental and simulated dissymmetry spectra for two different size samples. (e) Model chiral shape used in the simulation.

spectral shifts (for sizes of several nanometres), in the present case the relatively large size of the nanostructures rules out such effects, and as the DDA simulation confirms the size effects come from enhancement of absorption at wavelengths where electromagnetic cavity resonance modes occur. This, together with the shape chirality, turn the Te nanostructures shown in Figs 3 and 4 into a unique case of nanoscale chiral optical resonators.

The CD activity of the Te nanocrystals, observed both experimentally and theoretically, is due to the chiral shape of an absorbing and scattering object made of a semiconductor (Supplementary Fig. 10). This mechanism is somewhat different from the mechanisms leading to $\mathrm{CD}$ in molecules ${ }^{38,39}$ and also different from the case of metal nanostructures ${ }^{5-8}$. In metal nanostructures the $\mathrm{CD}$ has a plasmonic origin, whereas in $\mathrm{Te}$ nanocrystals the $\mathrm{CD}$ originates from inter-band exciton transitions as well as scattering of the large dielectric nanonanostructures with chiral shapes. Another finding out of the simulations is that the bisignate CD spectrum of a Te twister is relatively insensitive to its shape. Different right-handed shapes of a twister with the same overall size were simulated and always yielded CD spectra with positive short-wavelength part and a negative long-wavelength region (see Supplementary Fig. 11).

Transformation of chiral $\mathrm{Te}$ nanostructures into other materials. The reactivity of elemental $\mathrm{Te}$ to chemical transformations holds promise for templating of chiral nanostructures of various materials by depositing them on the chiral Te nanostructures. Here we demonstrate templating of chiral gold and silver telluride nanostructures. Many reports, such as by SanchezIglesias et $a .^{40}$, demonstrate templating of plasmonic gold particles by galvanic replacement of Te nanocrystals.

Transformation of $\mathrm{Te}$ nanostructures to silver telluride can also be achieved easily by reaction with silver ions ${ }^{41}$.
When nanocrystals of sample 5 were used as sacrificial templates, gold and silver telluride nanostructures with unique shapes were formed. TEM images of these particles together with their extinction and CD spectra are presented in Fig. 6 (see also material characterizations in Supplementary Figs 12-14). These transformations preserve the chiral shape very efficiently. For the gold nanostructures, an exceptionally large plasmonic CD with peak dissymmetry around $650 \mathrm{~nm}$ of $\sim 0.015$ was obtained. The two peaks seen in the absorption spectrum of this sample are the typical transverse $(\sim 550 \mathrm{~nm})$ and longitudinal $(\sim 900 \mathrm{~nm})$ resonances observed in elongated gold nanostructures. Thus, we see that the $C D$ peak extending from $600 \mathrm{~nm}$ onward into the infrared corresponds to the longitudinal plasmonic resonance, which is apparently more sensitive to the twist in the nanostructure than the transverse resonance. At the wavelength of the transverse resonance there is no noticeable CD signal. We may expect this, as the transverse plasmons create typically less absorption and exhibit much weaker CD.

\section{Discussion}

Colloidal nanostructures of Se and Te with various morphologies and large enantiomeric excess were prepared with the use of strongly binding chiral ligands and the extinction and CD spectra analysed. For Se samples, the $\mathrm{CD}$ is assigned to the intrinsic chirality of the crystal structure and the enantiomeric excess of nanocrystals formed in the synthesis. For Te, two hierarchies of chirality have been observed. For chiral lattice and achiral shape (nanorods), the dissymmetry value is small. For the case of chiral lattice and chiral shape (sample 5), the peak dissymmetry was very large. These observations were correlated with electromagnetic response simulations to prove that for Te the shape chirality is much more significant for visible wavelengths chiroptical activity than lattice chirality. Thus, the chiral Te 

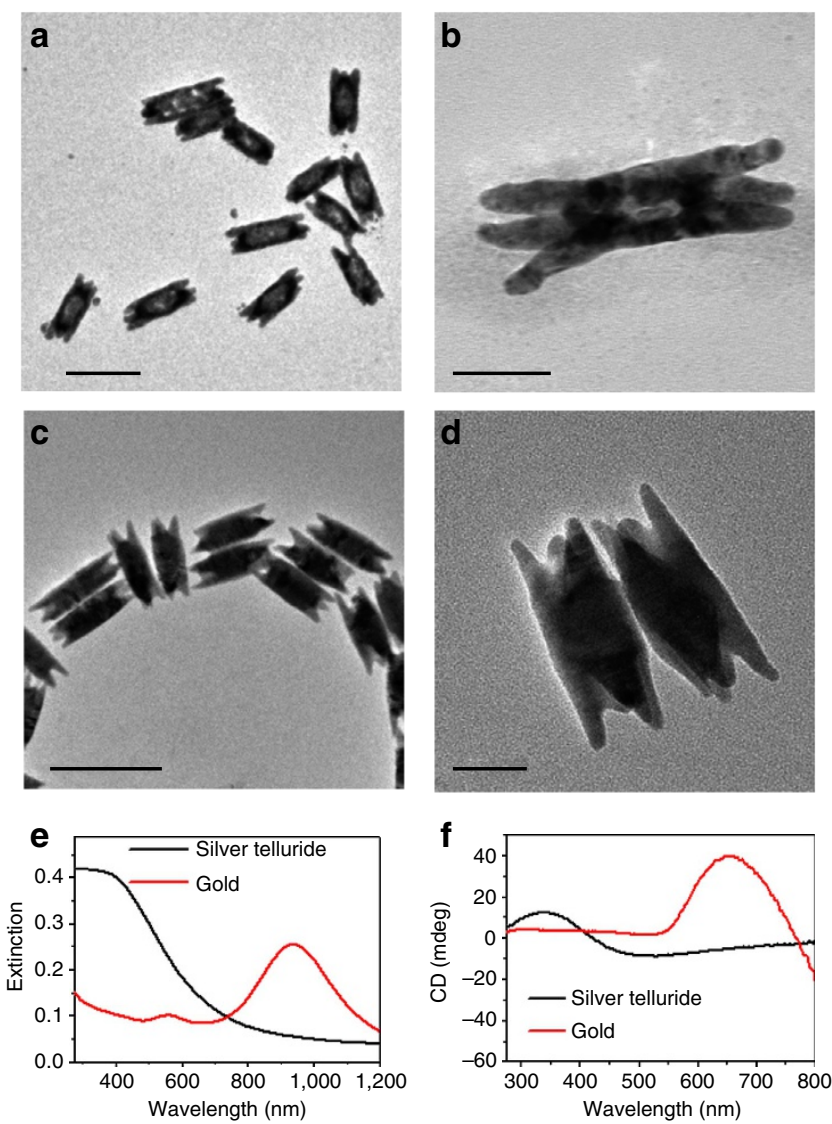

Figure 6 | Nanocrystals of gold and silver telluride formed by chemical transformation. (a,b) TEM images of such gold nanostructures. $(\mathbf{c}, \mathbf{d})$ TEM images of silver telluride nanocrystals. (e) Extinction and (f) CD spectra are depicted in the bottom row. Scale bars, $200 \mathrm{~nm}(\mathbf{a}, \mathbf{c})$ and $50 \mathrm{~nm}(\mathbf{b}, \mathbf{d})$.

nanostructures of size of the order of $100 \mathrm{~nm}$ act as chiral optical resonators, and might become useful in optical sensing of chiral molecules.

The results presented in this work prove that the concept of enantioselective synthesis of intrinsically chiral inorganic nanocrystals could be generalized to many material systems. A wide variety of chiral inorganic materials still remain for future studies, such as different crystals of $\mathrm{SiO}_{2}$ and $\mathrm{GeO}_{2}, \mathrm{AlPO}_{4}, \mathrm{Ag}_{2} \mathrm{Se}, \mathrm{CrSi}_{2}$, $\mathrm{HgO}, \mathrm{TeO}_{2}$ and more. Control over chirality in these systems may sometimes be not limited to lattice symmetry and may extend to the shape of the whole particle, making these systems intriguing for studies of chirality-associated effects at several size hierarchies. In this regard, the present work gives a first demonstration of mesoscale shape chirality for Te. Relation of shape to molecular scale chirality has been considered in the formation of chiral (twisted) mesoscopic shapes in several material systems ${ }^{42-44}$. For inorganic crystals, this has been observed in bulk form, in quartz for instance 45 . However, the exact mechanism of translation of molecular scale lattice chirality into whole crystal shape chirality is not fully established. We believe that the kind of material systems studied here are excellent candidates to test the generality of this concept and address the mechanisms involved.

It is expected that intrinsically chiral materials should be very useful for enantioselective chemistry (for which the lattice chirality is sufficient), as recently demonstrated by Soai and colleagues ${ }^{46}$ for asymmetric autocatalysis induced by macroscopic $\alpha-\mathrm{HgS}$ crystals. Nanocrystals should be even more efficient as catalysts due to much higher surface-to-volume ratio. In addition, recent reports indicate that materials composed of helical morphologies should be useful for spintronics applications ${ }^{47}$. On a fundamental level, these systems are expected to be intriguing candidates for studies of mechanisms acting in chiral crystallization, as well as studies of binding of organic molecules to chiral surfaces ${ }^{1,2}$, which is a key aspect in catalysis. An advantage of such studies is that the nano-colloidal systems are easy to monitor spectroscopically, as opposed to bulk solid-state samples where chiroptical spectroscopy is challenging ${ }^{48}$. One important message from this work is that although chiral crystals have been considered as sources for generation of homochirlity in biomolecules, the opposite effect of biomolecules inducing homochirality in crystals is also fascinating. The reactivity of Te towards chemical transformations might aid in future design of chiral nanostructure shapes of achiral materials, such as presented here for gold and silver telluride. Specifically, for the case of metallic systems with plasmon resonances, this method could be further developed for large-scale production of chiral meta-materials.

\section{Methods}

Chemicals. Tellurium dioxide $\left(\mathrm{TeO}_{2} ; 99.995 \%\right)$, selenous acid $(98 \%)$, gold chloride trihydrate $(\geq 99.9 \%)$, silver nitrate $(99.9 \%)$, hydrazine hydrate solution $(78-82 \%)$ L-glutathione (reduced form, $\geq 98 \%$ ), L-cysteine ( $\geq 98.5 \%$ ), D-cysteine ( $\geq 99 \%$ ), L-penicillamine (99\%), D-penicillamine ( $\geq 99 \%)$, L-arginine ( $\geq 98 \%)$, SDS (99\%), sodium borohydride $(98 \%)$ and sodium hydroxide $(97 \%)$ were purchased from Sigma and used without further purification. D-glutathione (purity according to HPLC 95\%) was obtained from Genscript USA, Inc. All water used was ultrapure $(18 \mathrm{M} \Omega \mathrm{cm})$, obtained from a USF ELGA UHQ system

Synthesis. For the biomolecule stock solutions, the reagent (glutathione, cysteine, penicillamine) was dissolved in water (final concentration $100 \mathrm{mM}$ ). Afterwards, the $\mathrm{pH}$ was adjusted to 11.5 using $\mathrm{NaOH}$ solution $(1 \mathrm{M})$.

For the $\mathrm{TeO}_{2}$ solution, $15 \mathrm{mg}$ of $\mathrm{TeO}_{2}$ was dispersed in $1 \mathrm{ml}$ of $20 \mathrm{mM} \mathrm{NaOH}$ aqueous solution. The material is only slightly soluble, and the solution remained turbid and was constantly stirred before and during the reaction.

All samples were prepared in $20 \mathrm{ml}$ glass vials, placed on a hot plate with magnetic stirring. To stop the reaction, $1 \mathrm{ml}$ of the sample was collected and diluted twice with $100 \mathrm{mM}$ SDS solution in water at room temperature.

Te nanocrystals (1). The temperature of the plate was set to $62^{\circ} \mathrm{C}$. The following reagents have been added in this order: $1 \mathrm{ml} \mathrm{TeO}_{2}$ solution, $1 \mathrm{ml} \mathrm{L}$-glutathione solution and $8 \mathrm{ml}$ water. The sample was collected after 10 min reaction.

Te nanorods (2). The temperature of the plate was set to $62^{\circ} \mathrm{C}$. The following reagents were added in this order: $1 \mathrm{ml} \mathrm{TeO}_{2}$ solution, $1 \mathrm{ml} \mathrm{L}$-glutathione solution and $5.5 \mathrm{ml}$ water were added (fast), followed by dropwise addition of $2.5 \mathrm{ml}$ hydrazine solution. Aliquots of the reaction have been removed every $10 \mathrm{~min}$.

Te nanocrystals ( 3 and 4). The temperature of the plate was set to $62^{\circ} \mathrm{C}$. The following reagents were added in this order: $1 \mathrm{ml} \mathrm{TeO}_{2}$ solution, $1 \mathrm{ml} \mathrm{L}$ - or Dpenicillamine solution and $5.5 \mathrm{ml}$ water were added (fast), followed by dropwise addition of $2.5 \mathrm{ml}$ hydrazine solution. The sample was obtained after $30 \mathrm{~min}$ of reaction.

Te nanocrystals (5). The following reagents were added in this order: $1 \mathrm{ml} \mathrm{TeO}_{2}$ solution, followed by $5.5 \mathrm{ml}$ water and dropwise addition of $2.5 \mathrm{ml}$ hydrazine solution. Finally $1 \mathrm{ml}$ glutathione ( $\mathrm{L}$ - or $\mathrm{D}$-form) solution was added. Aliquots were collected at different time intervals as described in the main text.

Se nanocrystals. To $2 \mathrm{ml}$ of water, $0.1 \mathrm{ml}$ aqueous solution of selenous acid $(100 \mathrm{mM})$ was added, followed by $0.1 \mathrm{ml}$ of hydrazine hydrate solution. After that, the $\mathrm{pH}$ was raised by addition of $0.02 \mathrm{ml} \mathrm{NaOH}$ solution $(1 \mathrm{M})$, and finally $0.1 \mathrm{ml}$ cysteine solution ( $\mathrm{L}$ - or D-form, $100 \mathrm{mM}$ in water) was added. The reaction was left for $24 \mathrm{~h}$ at room temperature in the dark. During that time the colour gradually evolved from yellow to orange to pink/purple. After that, the particles were collected by centrifugation and washed with water several times, followed by addition of $100 \mathrm{mM}$ SDS for stabilization.

Conversion of chiral Te nanostructures to gold. Sample $5(0.5 \mathrm{ml}$; diluted $\times 2$ with $100 \mathrm{mM}$ SDS solution) was purified by collecting the particles sediment after centrifugation, removing all the solution and dispersing in water (several times). 
After that, the particles were finally dissolved in $2 \mathrm{ml}$ water. A gold precursor solution containing $1 \mathrm{ml}$ of $100 \mathrm{mM} \mathrm{CTAB}$ solution, $0.2 \mathrm{ml} \mathrm{HAuCl}_{4}$ solution and $0.1 \mathrm{ml} \mathrm{NaOH}(1 \mathrm{M})$ solution was prepared. Of this solution, a quantity of $0.4 \mathrm{ml}$ was added to $2 \mathrm{ml}$ of purified sample 5 particles, and the reaction was left for $1 \mathrm{~h}$ before collecting the particles by centrifugation and washing with water several times.

Conversion of chiral Te nanostructures to silver telluride. Sample 5 (diluted $\times 2$ with $100 \mathrm{mM}$ SDS solution) was cleaned as described in the procedure of gold templating. Of the clean sample, $0.5 \mathrm{ml}$ were diluted with $1.5 \mathrm{ml}$ water and stirred vigorously. $\mathrm{AgNO}_{3}$ solution $(0.06 \mathrm{ml})$ in water $(100 \mathrm{mM})$ was added, and the solution was left to react for $1 \mathrm{~h}$. After that, particles were collected and cleaned by centrifugation.

Spectroscopic and structural characterization. CD measurements were performed using an Applied Photophysics Chirascan spectrometer. Absorption measurements were performed using a Varian Carry 5000 spectrophotometer. TEM imaging and diffraction was performed using a FEI Tecnai F20 FEG-TEM, with a gold nanoparticle standard used for calibration in diffraction. STEM imaging and tomography, as well as energy dispersive $\mathrm{X}$-ray spectroscopy measurements, were performed using a Tecnai F-20 S/TEM microscope (Twin lens) equipped with a Fischione Model 3000 high-angle annular dark-field detector.

Electron tomography. Tomographic data sets were obtained using Xplore3D software (FEI). Images were acquired in a tilt range of $\pm 70^{\circ}$, with $\mathrm{a}^{\circ}$ increment at the lower tilts (less than $46^{\circ}$ tilt) and a $1^{\circ}$ increment at the higher tilts. In total, 94 images were acquired. The images were aligned and reconstructed using a refinement procedure for the image alignment, which uses an iterative optimization of the tomogram resolution and SIRT algorithm for the subsequent tomogram reconstruction ${ }^{49}$. Three-dimensional visualization was performed by ImageVis3D freeware, version 3.0.0, developed at the University of Utah.

DDA calculations and model. The computational results given in the manuscript are based on a numerical solution of Maxwell's equations incorporating local dielectric functions of $\mathrm{Te}^{36}$ and water as a medium. $\left(\varepsilon_{\text {water }}=1.8\right)$ The Te crystal has intensive inter-band transitions that are reflected in the real and imaginary parts of the dielectric constant ${ }^{36}$. As a numerical method, we employed the $\mathrm{DDA}^{35,50}$ and the software from http://www.astro.princeton.edu/ draine/DDSCAT.html and ref. 51. CD is calculated as a difference of the optical extinctions for left and right circularly polarized (LCP and RCP) light beams: $\Delta \varepsilon=\varepsilon_{\mathrm{LCP}}-\varepsilon_{\mathrm{RCP}}$, where $\varepsilon_{\mathrm{LCP}}$ and $\varepsilon_{\mathrm{RCP}}$ are the extinction coefficients for the corresponding circularly polarized photons. Note that, in our calculations, the above extinction coefficients were averaged over the orientation of a nanocrystal relative to the direction of incident light ${ }^{52}$.

To mimic the Te nanocrystals synthesized in the project, we tried several shapes. The shape, which most closely resembles the experimental nanoparticles (Fig. 3), is shown in Fig. 5e. This shape is made of four merged nanorods. One wide nanorod is placed in the centre of the construction and the other three nanorods are attached to the central one. In addition, we created twisted stripes on the sides of the object and these stripes brought chirality to this geometry. In the first step, we created a mathematical function in the spherical coordinates, $\rho=f(\theta, \varphi)$, that defines the surface of such particle (Supplementary Fig. 10, inset). In the next step, we filled this geometrical object with point dipoles (Supplementary Fig. 15, inset). This is the model for the DDA computation.

Within the DDA approach, a crystalline object is represented as a set of point dipoles with certain polarizability and density values. When the number of dipoles is very large, the DDA model is reliable and describes very well optical properties of the initially continuous object. Here we apply this method for semiconductor nanocrystals with strong absorption and scattering. Supplementary Fig. 15 shows the reliability of our calculations. As an example, we consider the Te twister with the 140-nm length. We now vary the number of point dipoles in the DDA calculations, denoted as $N_{\text {dipoles }}$, by $\sim 8$ times and we do not observe significant changes in the calculated CD spectra. Our calculation results shown in Fig. $5 \mathrm{~b}, \mathrm{~d}$ and Supplementary Fig. 10 were done for $N_{\text {dipoles }}=1.5 \times 10^{5}$, whereas

Supplementary Fig. 15 depicts the data for three numbers $N_{\text {dipoles }}=6.5 \times 10^{4}$, $1.5 \times 10^{5}$ and $5.1 \times 10^{5}$.

\section{References}

1. Hazen, R. M. \& Sholl, D. S. Chiral selection on inorganic crystalline surfaces. Nat. Mater. 2, 367-374 (2003).

2. Weissbuch, I. \& Lahav, M. Crystalline architectures as templates of relevance to the origins of homochirality. Chem. Rev. 111, 3236-3267 (2011).

3. Berova, N., Polavarapu, P. L., Nakanishi, K. \& Woody, R. W. Comprehensive Chiroptical Spectroscopy (John Wiley and Sons Inc., 2012).

4. Valev, V. K., Baumberg, J. J., Sibilia, C. \& Verbiest, T. Chirality and chiroptical effects in plasmonic nanostructures: fundamentals, recent progress, and outlook. Adv. Mater. 25, 2517-2534 (2013).
5. Ben-Moshe, A., Maoz, B. M., Govorov, A. O. \& Markovich, G. Chirality and chiroptical effects in inorganic nanocrystal systems with plasmon and exciton resonances. Chem. Soc. Rev. 42, 7028-7041 (2013).

6. Govorov, A. O. et al. Chiral nanoparticle assemblies: circular dichroism, plasmonic interactions, and exciton effects. J. Mater. Chem. 21, 16806-16818 (2011).

7. Govorov, A. O., Fan, Z., Hernandez, P., Slocik, J. M. \& Naik, R. R. Theory of circular dichroism of nanomaterials comprising chiral molecules and nanocrystals: plasmon enhancement, dipole interactions, and dielectric effects. Nano Lett. 10, 1374-1382 (2010).

8. Govorov, A. O. \& Fan, Z. Theory of chiral plasmonic nanostructures comprising metal nanocrystals and chiral molecular media. Chem. Phys. Chem 13, 2551-2560 (2012).

9. Abdulrahman, N. A. et al. Induced chirality through electromagnetic coupling between chiral molecular layers and plasmonic nanostructures. Nano Lett. 12, 977-983 (2012).

10. Lieberman, I., Shemer, G., Fried, T., Kosower, E. M. \& Markovich, G. Plasmonresonance-enhanced absorption and circular dichroism. Angew. Chem. Int. Ed. 47, 4855-4857 (2008).

11. Melnikau, D., Savateeva, D., Gun'ko, Y. K. \& Rakovich, Y. P. Strong enhancement of circular dichroism in a hybrid material consisting of J-aggregates and silver nanoparticles. J. Phys. Chem. C 117, 13708-13712 (2013).

12. Maoz, B. M. et al. Amplification of chiroptical activity of chiral biomolecules by surface plasmons. Nano Lett. 13, 1203-1209 (2013).

13. Guerrero-Martínez, B. et al. Intense optical activity from three-dimensional chiral ordering of plasmonic nanoantennas. Angew. Chem. Int. Ed. 50, 5499-5503 (2011).

14. Hentschel, M., Schäferling, M., Weiss, T., Liu, N. \& Giessen, H. Threedimensional chiral plasmonic oligomers. Nano Lett. 12, 2542-2547 (2012).

15. Tang, Y., Sun, L. \& Cohen, A. E. Chiroptical hot spots in twisted nanowire plasmonic oscillators. Appl. Phys. Lett. 102, 0431031-0431034 (2013).

16. Yan, W. et al. Self-assembly of chiral nanoparticle pyramids with strong R/S optical activity. J. Am. Chem. Soc. 134, 15114-15121 (2012).

17. Ma, W. et al. Chiral plasmonics of self-assembled nanorod dimers. Sci. Rep. 3, 1934 (2013).

18. Soukoulis, C. M. \& Wegener, M. Past achievements and future challenges in the development of three-dimensional photonic metamaterials. Nat. Photon. 5, 523-530 (2011).

19. Hendry, E. et al. Ultrasensitive detection and characterization of biomolecules using superchiral fields. Nat. Nanotech. 5, 783-787 (2010).

20. Frank, B. et al. Large-area 3D chiral plasmonic structures. ACS Nano 7, 6321-6329 (2013).

21. Kuzyk, A. et al. DNA-based self-assembly of chiral plasmonic nanostructures with tailored optical response. Nature 483, 311-314 (2012).

22. Mark, A. G., Gibbs, J. G., Lee, T.-C. \& Fischer, P. Hybrid nanocolloids with programmed three-dimensional shape and material composition. Nat. Mater. 12, 802-807 (2013).

23. Yeom, B. et al. Chiral plasmonic nanostructures on achiral nanopillars. Nano Lett. 13, 5277-5283 (2013).

24. Liu, S. et al. Synthesis of chiral $\mathrm{TiO}_{2}$ nanofibre with electron transition-based optical activity. Nat. Commun. 3, 1215 (2012).

25. Che, S. et al. Synthesis and characterization of chiral mesoporous silica. Nature 429, 281-284 (2004).

26. Shopsowitz, K. E., Qi, H., Hamad, W. Y. \& MacLachlan, M. J. Free-standing mesoporous silica films with tunable chiral nematic structures. Nature 468, 422-425 (2010).

27. Saeva, F. D., Olin, G. R. \& Chu, J. Y. C. Circular dichroism of trigonal selenium formed in a chiral polymer matrix. Mol. Cryst. Liq. Cryst. 41, 5-9 (1977).

28. Ben-Moshe, A., Govorov, A. O. \& Markovich, G. Enantioselective synthesis of intrinsically chiral mercury sulfide nanocrystals. Angew. Chem. Int. Ed. 52, 1275-1279 (2013).

29. Mayers, B. \& Xia, Y. Formation of tellurium nanotubes through concentration depletion at the surfaces of seeds. Adv. Mater. 14, 279-282 (2002).

30. Gates, B., Mayers, B., Cattle, B. \& Xia, Y. Synthesis and characterization of uniform nanowires of trigonal selenium. Adv. Mater. 12, 219-227 (2002).

31. Tang, Z., Wang, Y., Sun, K. \& Kotov, N. A. Spontaneous transformation of stabilizer-depleted binary semiconductor nanoparticles into selenium and tellurium nanowires. Adv. Mater. 17, 358-363 (2005).

32. Albeck, A., Weitman, H., Sredni, B. \& Albeck, M. Tellurium compounds: selective inhibition of cysteine proteases and model reaction with thiols. Inorg. Chem. 37, 1704-1712 (1998).

33. Sreeprasad, T. S., Samal, A. K. \& Pradeep, T. Bending and shell formation of tellurium nanowires induced by thiols. Chem. Mater. 21, 4527-4540 (2009).

34. Viedma, C., McBride, J. M., Kahr, B. \& Cintas, P. Enantiomer-specific oriented attachment: formation of macroscopic homochiral crystal aggregates from a racemic system. Angew. Chem. Int. Ed. 52, 10545-10548 (2013). 
35. Flatau, P. J. \& Draine, B. T. Fast near field calculations in the discrete dipole approximation for regular rectilinear grids. Opt. Express 20, 1247-1252 (2012). 36. Palik, E. D. Handbook of Optical Constants of Solids (Academic, 1985).

37. Fan, Z., Zhang, H. \& Govorov, A. O. Optical properties of chiral plasmonic tetramers: circular dichroism and multipole effects. J. Phys. Chem. C 117, 14770-14777 (2013)

38. Barron, L. D. Molecular Light Scattering and Optical Activity 2nd edn (Cambridge University Press, 2004).

39. Bustamante, C., Tinoco, Jr I. \& Maestre, M. F. Circular differential scattering can be an important part of the circular dichroism of macromolecules. Proc. Natl Acad. Sci. USA 80, 3568-3572 (1983).

40. Sanchez-Iglesias, A. et al. Gold colloids with unconventional angled shapes. Langmuir 25, 11431-11435 (2009)

41. Moon, G. D., Ko, S., Xia, Y. \& Jeong, U. Chemical transformations in ultrathin chalcogenide nanowires. ACS Nano 4, 2307-2319 (2010).

42. Oda, R., Huc, I., Schmutz, M., Candau, S. J. \& Mackintosh, F. C. Tuning bilayer twist using chiral counterions. Nature 399, 566-569 (1999).

43. Li, C. Y. et al. Double twist in helical polymer 'soft' crystals. Phys. Rev. Lett. 83, 4558-4561 (1999).

44. Li, C. Y. et al. Left or right, it is a matter of one methylene unit. J. Am. Chem. Soc. 123, 2462-2463 (2001).

45. Shtukenberg, A. G., Punin, Y. O., Guyral, A. \& Kahr, B. Growth actuated bending and twisting of single crystals. Angew. Chem. Int. Ed. 53, 672-699 (2014).

46. Shindo, H. et al. Asymmetric autocatalysis induced by cinnabar: observation of the enantioselective adsorption of a 5-pyrimidyl alkanol on the crystal surface. Angew. Chem. Int. Ed. 52, 9135-9138 (2013).

47. Göhler, B. et al. Spin selectivity in electron transmission through self-assembled monolayers of double-stranded DNA. Science 331, 894-897 (2011).

48. Freudenthal, J. H., Hollis, E. \& Kahr, B. Imaging chiroptical artifacts. Chirality 21, E20-E27 (2009).

49. Houben, L. \& Bar-Sadan, M. Refinement procedures for the image alignment in high-resolution electron tomography. Ultramicroscopy 111, 1512 (2011).

50. Draine, B. T. \& Flatau, P. J. Discrete-dipole approximation for scattering calculations. J. Opt. Soc. Am. A 11, 1491-1499 (1994).

51. Draine, B. T. \& Flatau, P. J. User Guide for the Discrete Dipole Approximation Code DDSCAT 7.2. Preprint at http://arXiv.org/abs/1202.3424 (2012).
52. Fan, Z. \& Govorov, A. O. Chiral nanocrystals: plasmonic spectra and circular dichroism. Nano Lett. 12, 3283-3289 (2012).

\section{Acknowledgements}

This research was supported by The Israel Science Foundation grant no. 172/10, the James Frank programme on light-matter interaction. A.B.-M. is supported by the Adams Fellowship Program of the Israel Academy of Sciences and Humanities. Electron microscopy was performed at the Wolfson Applied Materials Research Centre at Tel Aviv University and at the Irving and Cherna Moskowitz Center for Nano and Bio-Nano Imaging at the Weizmann Institute of Science. Z.F. and A.O.G. were supported by Volkswagen Foundation (Germany). Use of the Center for Nanoscale Materials was supported by the U.S. Department of Energy, Office of Science, Office of Basic Energy Sciences, under Contract Number DEAC02-06CH11357. The help of Dr Ronit PopovitzBiro in obtaining the energy dispersive X-ray spectroscopy scan lines is gratefully acknowledged.

\section{Author contributions}

A.B.-M. initiated the study, conceived and carried out the syntheses and the characterizations, and analysed the data. S.G.W. did the acquisition of the STEM tomography images. M.B.S. and L.H. did the electron tomography 3 D reconstructions. Z.F. and A.O.G. constructed the geometrical twister model, performed the DDA simulations and helped writing the discussion. G.M. supervised the experiments and wrote the paper together with A.B.M.

\section{Additional information}

Supplementary Information accompanies this paper at http://www.nature.com/ naturecommunications

Competing financial interests: The authors declare no competing financial interests.

Reprints and permission information is available online at http://npg.nature.com/ reprintsandpermissions/

How to cite this article: Ben-Moshe, A. et al. Enantioselective control of lattice and shape chirality in inorganic nanostructures using chiral biomolecules. Nat. Commun 5:4302 doi: 10.1038/ncomms5302 (2014). 